of free space (to a close approximation, equal to that of the patient/air within an MRI scanner).

This simple formula works out at approximately $1 \times 10^{6}$ ampere-turns (around 10,000 turns of wire driven with $100 \mathrm{amps}$ ) for a typical MRI magnet length of 1.2 metres and a field of 1 tesla. For several reasons, practical MRI magnets actually have around 5 times as many ampereturns. Firstly because of their small length-to-diameter ratio; secondly because of the increasing turns density needed towards the ends of the coil to make the field homogeneous; and lastly because of the need for active stray-field compensation (the magnet consists of two concentric coil sets with opposing polarity, shaped in such a way that the outer coil to a large extent cancels the external magnetic field of the inner coil).

Without the use of superconducting materials such as Niobium Titanium, cooled with liquid helium to below their critical temperature, the energy loss in such coils would make modern MRI scanners impractical. Thanks to these materials, however, MRI scanners are already commonplace in hospitals, providing superior soft-tissue imaging to assist doctors in the diagnosis of a wide range of diseases, including cancer, Alzheimer's, stroke, trauma injury and Parkinson's disease.

\section{Field strength and uniformity}

The characteristics of the magnetic field generated by an MRI scanner have a significant impact on its performance. The field strength has to be very uniform in the imaging volume (typically the cross-section of the human body, with a spherical volume of around 45 to $50 \mathrm{~cm}$ diameter in the center of the magnet) and it has to remain extremely stable during the scan. In addition, the proton polarization, which determines the strength of the NMR signal, increases linearly with field strength. High field strengths therefore enable increased signalto-noise ratios and better image quality. They also allow shorter scan times, increasing patient throughput and reducing patient discomfort. There is therefore a trend toward higher field strength scanners, with 3T MRI scanners already being used in routine clinical care and 7T machines being used in (pre-)clinical research.

However, 7T MRI scanners are extremely expensive and will probably remain so, in particular because the currentcarrying capacity of the superconductors employed decreases rapidly above $5 \mathrm{~T}$, leading to a disproportionate increase in the amount of conductor needed. In addition, the electro-magnetic (Lorentz) forces that have to be contained become a real problem, increasing with the square of the field. The main problem with Lorentz forces on superconducting conductors is that they can lead to motion of the conductors, which causes friction heat. It only takes a few microjoules of heat energy to raise a small piece of the superconductor above its critical temperature (the heat capacity at $4 \mathrm{~K}$ is extremely small) and if this non-superconducting zone starts to propagate through the coil, the entire stored energy of the magnet (many megajoules) is dumped into the liquid helium cooling the magnet (this is known as a quench). MRI magnets are designed to survive such an event without permanent damage, but re-cooling and bringing the magnet back to the required field strength is time consuming and costly.

\section{Cryostat design}

In most of today's MRI magnets, the superconducting coil is enclosed in a helium tank containing approximately 1000 litres of liquid when full. Magnets are usually cooled down and filled only once, in the factory where they are made, and remain cold for their entire life (typically 10 years).

\section{HOW MRI WORKS}

When a person is in an MRI scanner, the hydrogen nuclei (i.e., protons, found in abundance in the human body as water) align with the strong magnetic field. A radio wave at just the right frequency for the protons to absorb energy pushes some of the protons out of alignment. The protons then snap back to alignment, producing a detectable rotating magnetic field as they do so. Since protons in different areas of the body (e.g., fat versus muscle) realign at different speeds, the different structures of the body can be revealed.
Gradient fields in the three dimensions allow the scanner to work only with protons from a 'slice' at a time, allowing the creation of a whole volume that can be looked at in three dimensions.

Contrast agents may be injected intravenously to show enhancement of blood vessels, tumors or inflammation. Unlike CT scanning, MRI uses no ionizing radiation and is generally a very safe procedure. Patients with some metal implants and cardiac pacemakers are prevented from having an MRI due to effects of the powerful magnetic field and powerful radio waves.

MRI is used to image every part of the body, but is particularly useful in neurological conditions, disorders of the muscles and joints, for evaluating tumors and showing abnormalities in the heart and blood vessels.

\section{source:}

www.websters-online-dictionary.org/ definitions/Magnetic+resonance+ imaging 
Any heat leak into this cold mass causes helium to evaporate (at a rate of around 1.4 litres per hour per watt of thermal load). If the cold mass is enclosed in a

\section{INCREASED PATIENT COMFORT AND ACCESS}

Some patients feel very claustrophobic in the bore of a conventional cylindrical magnet MRI scanner, and the sudden loud noises emitted by the scanner when the gradient coils are activated often worsens this feeling and can cause the patient to move suddenly. Such movements can degrade image quality, so sedatives and/or ear plugs are often used to calm the patient and minimize the effect.

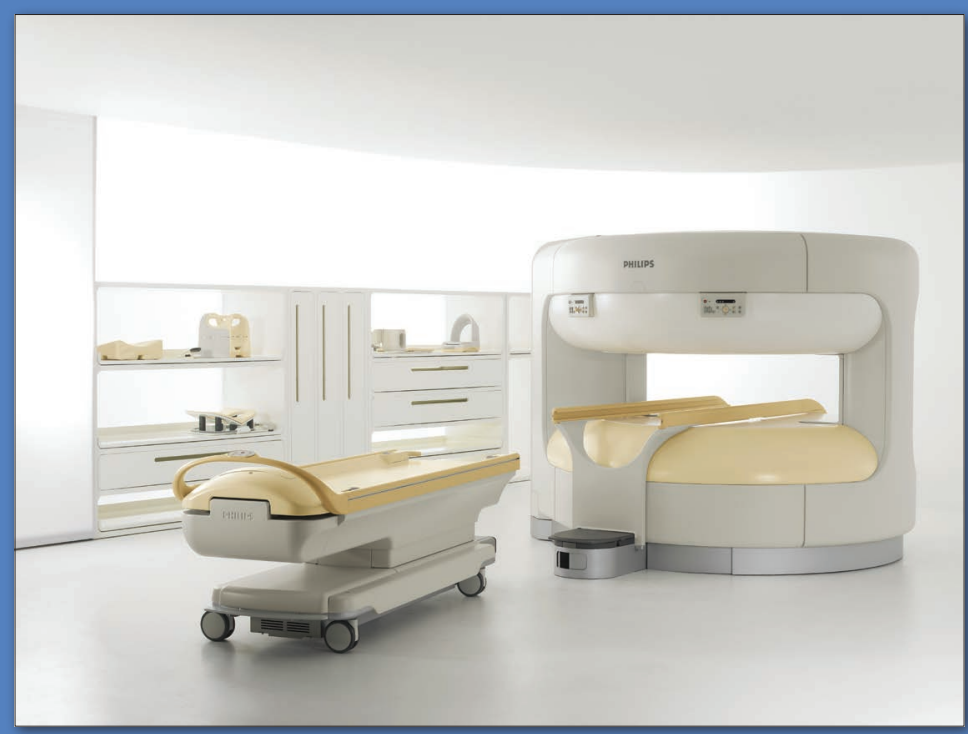

$\triangle$ The Panorama HFO, Philips' high-field, wide open system, offers outstanding dinical versatility with leading solutions for all clinical applications. And it is preferred by patients because of its comfortable space and wide open bore.

In an attempt to increase patient comfort and provide clinicians with better access to the patient, for example to perform MRI-guided clinical interventions, open-magnet MRI scanners have been developed. However, such designs are very challenging, since the C-shaped magnets used in these scanners are less efficient than cylindrical magnets; the mechanical forces generated are less symmetrical and therefore more difficult to control; and the split cryostat required becomes more complicated. Field strengths much in excess of 1T are therefore impractical. However, because open-magnet designs facilitate better receive-coil layouts, a 1 T open-magnet scanner such as the Philips Panorama 1.0T illustrated above can achieve the same imaging quality as a 1.5T cylindrical magnet design. .

v The Panorama HFO's open design provides space for dynamicimaging of joints in different positions.

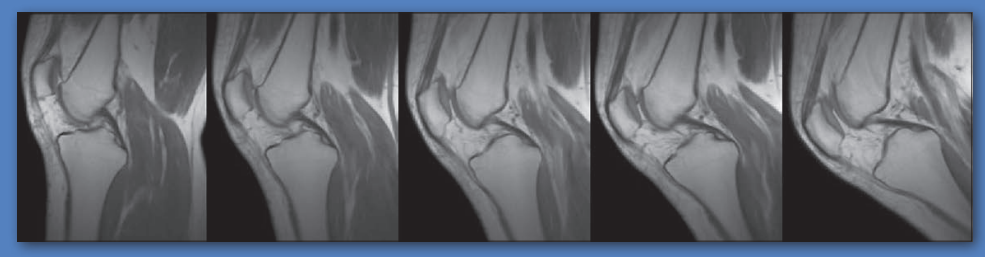

high-vacuum space and the magnet's suspension system has a low thermal conductivity, the single biggest contributor to this heat load is thermal radiation. Older MRI magnets relied on two refrigerator-cooled shields in the vacuum space to reduce the heat load to less than 50 $\mathrm{mW}$ (the radiated heat decreases with the fourth power of the surface temperature of the shield seen by the cold mass), resulting in a loss of less than 1 litre of helium per day. More modern refrigerators have about $1 \mathrm{~W}$ of cooling power at $4 \mathrm{~K}$, allowing boil-off from the helium tank to be stopped completely. These zero boil-off magnets are now the state of the art. The rapidly increasing cost of helium more than justifies the extra cost of such a refrigerator. However, it takes about $7 \mathrm{~kW}$ of electrical power to drive the refrigeration system.

One of the challenges in magnet design is therefore to make these refrigeration systems more efficient and reliable so that the loss of helium due to planned and un-planned downtime of the refrigeration system is minimized. When the magnet is being shipped or temporarily stored before installation, the refrigerator is also kept running whenever possible.

With the escalating cost of helium, which is a by-product of natural-gas extraction, another important challenge for the future is minimizing the helium content of the magnet. A few hundred litres of liquid helium or less would actually be sufficient to keep the magnet superconducting, so the main challenge in developing magnets without a large helium buffer volume is to make the refrigeration system so reliable that it never fails, or so efficient that it can re-cool the magnet without using much external helium after a failure.

\section{High-temperature superconductors}

One way of eliminating the need for helium in MRI scanners completely would be to use high-temperature superconducting materials such as Yttrium Barium Copper Oxide (YBCO) tape. This material remains superconducting at temperatures above $77 \mathrm{~K}$, although for a practical magnet an operating temperature of about $40 \mathrm{~K}$ to $50 \mathrm{~K}$ would be ideal. Being able to run the magnet at $40 \mathrm{~K}$ to $50 \mathrm{~K}$ instead of $4 \mathrm{~K}$ would allow a simpler and therefore more reliable refrigerator to be used, and the electrical energy required to keep the magnet cool could probably be reduced to around $1 \mathrm{~kW}$. The problem, however, is that YBCO tape is currently 200 to 300 times more expensive than Niobium Titanium wire, which means that its use in MRI scanners does not at the moment make economic sense.

\section{Clinical applications}

Challenges may still lie ahead for the future of MRI, but the good thing is that in forty years it has turned from an experimental device to a highly effective tool for the diagnosis of disease. Compared to other commonly used 
imaging modalities such as X-ray CT, it provides far superior soft-tissue imaging and poses no threat to patients or operators in terms of exposure to potentially harmful ionizing radiation.

Despite this, one of the early expectations for the Nuclear Magnetic Resonance (NMR) principles on which MRI is based has still not been realized. MRI can visualize lesions deep within the human body, but it cannot on its own determine whether or not they are malignant. Differential diagnosis still has to be done by biopsy. Nevertheless, advances are being made that could allow MRI to reveal some of the functional characteristics of malignant tumors, turning it from a purely anatomical imaging modality into a functional imaging modality. MRI is already being used as an imaging tool to perform brain scans, where its ability to generate contrast images that distinguish oxygen-rich blood from oxygen-depleted blood is used to spatially localize brain metabolism. This same ability is also being used to provide an indirect measurement of the angiogenesis (the proliferation of blood capillaries) and increased tissue metabolism associated with malignant tumors. Contrast agents, based on biocompatible paramagnetic materials such as chelated organic gadolinium complexes, that are injected into the blood stream, are another means of using MRI to functionally image vascular structures. Enhancing this procedure using antibody complexes that bind specifically to the surface of tumor cells could also allow paramagnetic molecules to be used to highlight tumors.

Since diagnoses based on functional imaging often require the capture of dynamic behaviour, functional MR imaging could benefit from the use of scanners with high temporal resolution, i.e., short image volume capture times. Unfortunately, the physics of MRI scanners means that spatial resolution and temporal resolution have to be traded off against one another. One way of achieving both is to move to higher magnetic field strengths. Despite their higher cost, 7T scanners may therefore find clinical applications in specialist diagnostic centers once appropriate clinical approvals are granted. At the moment they are only approved for research applications, typically with a focus on cardiac, oncology and neuro applications. Because of their excellent soft tissue imaging and the absence of ionizing radiation, MRI scanners could also provide clinicians with the real-time imaging needed for minimally invasive interventional procedures such as catheter-based heart valve replacement. However, they would preferably need to be open-magnet designs that provide clinicians with unhindered access to the patient. Philips is already collaborating with researchers at the University Medical Center Utrecht (Utrecht, The Netherlands) to combine an MRI scanner with a linear accelerator in order to investigate the benefits of MRIguided radiotherapy.
Despite the fact that the technology underlying MRI scanners is relatively mature, with the result that they are now routinely used in radiology, there is clearly much that can still be done to exploit the full potential of this important imaging modality. The quest for higher resolution images, better temporal resolution and improved functional imaging will continue to resolve unmet clinical needs in imaging-based diagnostics. .

\section{About the Authors}

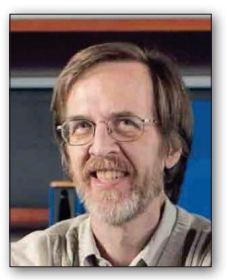

Johan Overweg is Principal Scientist at Philips Research in Hamburg, Germany, for MR front-end hardware, and leader of several projects. He studied Technical Physics at Twente University in Enschede, the Netherlands, and graduated in 1978 in low-temperature physics. He obtained his $\mathrm{PhD}$ at Twente University in 1982 with a thesis on ultra-sensitive dynamic magnetization measurements on paramagnetic substances at low temperature.

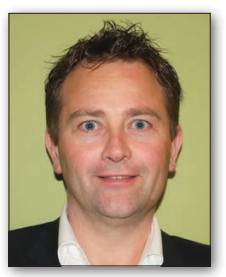

René Aarnink, MSc, $\mathrm{PhD}$, is leading the department for Minimally Invasive Healthcare at Philips Research in Eindhoven, the Netherlands. This department drives the developments of minimally invasive approaches for diagnostic and therapeutic medical devices for the professional healthcare market. Prior to joining Philips Research, René led clinical science departments in Philips Healthcare for MRI, X-ray and CT. He has a Master of Science degree in Electrical Engineering from University of Twente in Enschede, the Netherlands and $\mathrm{PhD}$ in Medical Science from the University of Nijmegen, the Netherlands. He (co-)authored more than 50 publications, conference papers and book chapters.

\section{LOW-FIELD-STRENGTH MRI}

There are some applications where low field strengths could be an advantage. For example, patients with implants that contain ferromagnetic materials cannot currently be placed in an MRI scanner. The proton relaxation modes in low field strengths can also generate some interesting image contrasts. The main problem at low field strengths is that the RF signal generated by nuclear magnetic resonance is weak and the signal-to-noise ratio is therefore poor. One way of overcoming this could be to pre-polarize the protons rather than relying only on the magnetic field to do it. The transfer of nuclear spin polarization from one nuclear spin population to another via cross-relaxation (the Overhauser Effect) is being investigated, but because one spin population would probably need to be free electrons, which act in the body like free-radicals, biocompatibility could be a major problem. Research is also being done on contrast agents that can be pre-polarized before injection. However, the required pre-polarization is difficult to produce and its lifetime is only a few minutes. 